\title{
Numerical investigation of convective heat transfer in a plane channel filled with metal foam under local thermal non-equilibrium
}

\author{
A. Settar ${ }^{1,2, a}$, R. Nebbali $^{1}$, B. Madani $^{1}$ And SaÏd Abboudi ${ }^{2}$ \\ 1 USTHB- Faculty of Mechanical Engineering and Process Engineering LTPMP, BP 32 El Alia, 16111 Bab Ezzouar Algiers, \\ Algeria \\ 2 Institut IRTES-M3M, EA 7274, UTBM site de Sévenans, 90010 Belfort Cedex, France
}

Received 17 April 2014, Accepted 18 December 2014

\begin{abstract}
The present work consists on convective heat transfer modeling in a plate heat exchanger filled with metal foam under local thermal non-equilibrium (LTNE). The metal foam was inserted to fill completely the studied channel, which is crossed by a fluid. The modified Brinkman-Forchheimer extended Darcy model is used in the porous layer, while the macroscopic two-energy equation model is used for the thermal field. The channel walls are maintained at a constant temperature and the velocity at the inlet is supposed uniform. A dimensionless formulation is developed to perform a parametric study in terms of certain dimensionless variables, and solved by the finite volume method (FVM). The results include the effect of the interstitial heat transfer coefficient and the solid to fluid thermal conductivity of different type of metal foams. The results were used to estimate the influence of the convective and conductive contributions using open-celled metal foams with high porosity. It has been shown that such supports can bring a significant enhancement for the heat transfer.
\end{abstract}

Key words: Convective heat transfer / two-equation model / metal foam / local thermal non-equilibrium / plate heat exchanger

\section{Introduction}

Metal foams are a type of porous materials with novel thermal, electrical and chemical properties which have considerable industrial applications, such as catalyst carriers, filters, biomedical applications, and spacecraft [1-3]. The use of metal foams in heat exchangers and chemical applications can provide evident advantages due to their high specific interfacial area, their high thermal conductivity, low density and a good ability of mixing fluid (Fig. 1). Thus, the open cell metal foams may substantially enhance the heat transfer process into heat exchangers. The metal foams can also be used as catalyst support with superior heat and mass transfer due to their optimal thermal properties. Compared to the conventional heat exchangers, the performance of those filled with open-celled metal foams can be enhanced due to the increased fluid/solid contact specific area. The probability of the remarkable temperature gradients resulting in the occurrence of the hot spots, cold zones and decreasing the device efficiency is minimized. Although, trans-

\footnotetext{
${ }^{a}$ Corresponding author: settarhakim@gmail.com
}

port phenomena in porous media are studied for nearly two centuries, the studies about metal foams are still relatively few and recent [4-7]. Thus, the study of heat transfer by forced convection in metal foams has been the subject of many studies [8-10], most of these works focus on the assumption of local thermodynamic equilibrium (LTE) [11-13]. In certain configurations using metal foams, where the solid to fluid thermal conductivity is high, the net heat transfer between phases takes place such that the assumption of LTE is not satisfied. The need for two-energy equation model becomes necessary. In the last years, the problem of LTNE has received considerable attention due to its presence in a wide variety of engineering applications, especially when metal foams are used for the enhancement process. This fact is confirmed by the high amount of studies that focused on comprehension of behavior of metal foam based on the LTNE assumption, in particular, the plane channel configuration filled with this porous medium $[1,14]$ because of its direct applicability in the plate heat exchangers and heat sinks. Zhao and $\mathrm{Lu}[8]$ presented an analytical solution with two-equation model for a heat exchanger filled with 


\section{Nomenclature}

\begin{tabular}{|c|c|c|c|}
\hline \multicolumn{2}{|r|}{ Latin characters } & $\bar{U}$ & Dimensionless vilocity $[-]$ \\
\hline$a_{s f}$ & Specific interfacial area $\left[\mathrm{m}^{-1}\right]$ & $u$ & Local velocity $\left[\mathrm{m} \cdot \mathrm{s}^{-1}\right]$ \\
\hline$B i$ & Interstitial Biot number $[-]$ & $\bar{u}_{D}$ & Iintrinsic volume average velocity $\left[\mathrm{m} . \mathrm{s}^{-1}\right]$ \\
\hline$c_{f}$ & Inertial factor (Forchheimer coefficient) $[-]$ & $V$ & Dimensionless velocity $[-]$ \\
\hline$c p$ & Fluid specific heat $\left[\mathrm{J}^{\mathrm{kg}}{ }^{-1} \cdot \mathrm{K}^{-1}\right]$ & $Y$ & Dimensionless transversal coordinate $[-]$ \\
\hline$d_{f}$ & Fiber diameter $[\mathrm{m}]$ & $X$ & Dimensionless longitudinal coordinate $[-]$ \\
\hline$d_{p}$ & Pore diameter $[\mathrm{m}]$ & & Greek characters \\
\hline$D a$ & Darcy number $[\mathrm{m}]$ & $\mu$ & Fluid dynamic viscosity $[\mathrm{Pa} . \mathrm{s}]$ \\
\hline$h_{s f}$ & Interstitial heat transfer coefficient $\left[\mathrm{W} \cdot \mathrm{m}^{-2} \cdot \mathrm{K}^{-1}\right]$ & $\rho$ & Fluid density $\left[\mathrm{kg} \cdot \mathrm{m}^{-3}\right]$ \\
\hline$H$ & Channel height [m] & $\omega$ & Pore density [PPI] (pore per inch) \\
\hline$K$ & Permeability $\left[\mathrm{m}^{-2}\right]$ & $\varepsilon$ & Porosity $[-]$ \\
\hline$k_{f}$ & Fluid thermal conductivity $\left[\mathrm{W} \cdot \mathrm{m}^{-2} \cdot \mathrm{K}^{-1}\right]$ & $\theta$ & Dimensionless local temperature [-] \\
\hline$k_{s}$ & Solid thermal conductivity $\left[\mathrm{W} \cdot \mathrm{m}^{-2} \cdot \mathrm{K}^{-1}\right]$ & $\Delta T_{r e f}$ & Reference difference temperature $[\mathrm{K}]$ \\
\hline$L$ & Channel length $[\mathrm{m}]$ & & Subscripts \\
\hline$p$ & Pressure $[\mathrm{Pa}]$ & $\overline{e f f}$ & Effective variable \\
\hline$P$ & Dimensionless pressure [-] & $f$ & Fluid phase \\
\hline $\operatorname{Pr}$ & Prandtl number $[-]$ & $s$ & Solid phase \\
\hline$R e$ & Reynolds number [-] & $w$ & Wall \\
\hline$R e_{d}$ & Reynolds number based on D and intrinsic & 0 & Inlet \\
\hline & velocity $\bar{u}_{D}[-]$ & & Other symbol \\
\hline$R_{k}$ & Solid to fluid thermal conductivity ratio [-] & $\phi$ & Local volume average of a quantity $\phi$ \\
\hline$T$ & Temperature $[\mathrm{K}]$ & $|\vec{V}|$ & Magnitude of the vector velocity $\vec{V}$ \\
\hline
\end{tabular}

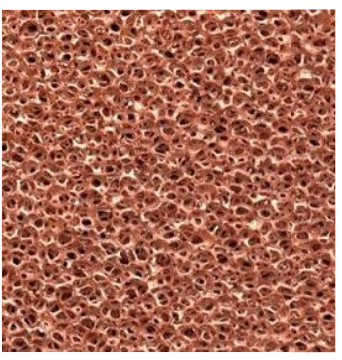

(a)

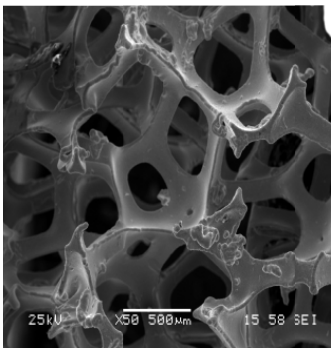

(b)

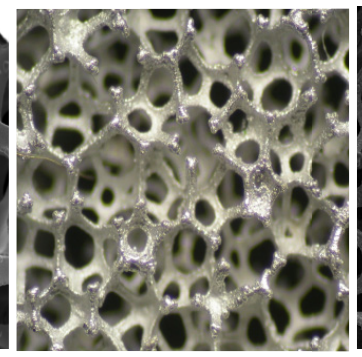

(c)

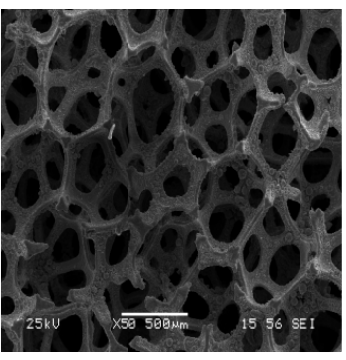

(d)

Fig. 1. Metal foams of different materials: (a) Copper, (b) Nickel, (c) Aluminum, (d) FrCrAlloy.

metal foam. They have examined the effect of morphology on the heat transfer. Their study showed that the heat exchangers filled with metal foams are more efficient than those with smooth tubes. Kim et al. [15] studied the effect of parameters such as the Darcy, Prandtl and Reynolds numbers in porous media. They showed that LTE assumption is valid when the convection heat transfer is dominant. Vafai and Kim [9] adopted the LTE assumption and conducted an analytical study of heat transfer by forced convection in porous media. Lee and Vafai [16] presented analytical solution for the parallel plates by considering the two-equation model. Similarly, Dukhan [17] conducted an analytical study of forced convection in a porous medium based on the LTNE assumption.

The aim of this work is to study the dynamic and thermal behaviors of a plate heat exchanger provided with a metal foam. We present a numerical investigation of forced convection into a flat channel completely filled with metallic foam. The work is particularly focused in the thermal field by adopting the two-equation model. The results according to this assumption are analyzed and discussed, highlighting the effect of various heat transfer parameters. In this study, the dynamic field is obtained using the general model of Darcy-Brinkman-Forchheimer.

\section{Physical and mathematical models}

\subsection{Physical model}

The schematic diagram of the problem is presented in Figure 2: flow in a channel, of length $L$ and height $H$, completely filled with porous material type metal foam and heated with a constant and uniform wall temperature $T_{w}$. The flow is assumed to be steady, incompressible, laminar and two-dimensional. A stream of air with uniform velocity $U_{0}$ and temperature $T_{0}$ is considered at the inlet of the channel. For the thermal field, the thermophysical properties are considered constant and the effects of natural convection and the viscous dissipation are neglected. 


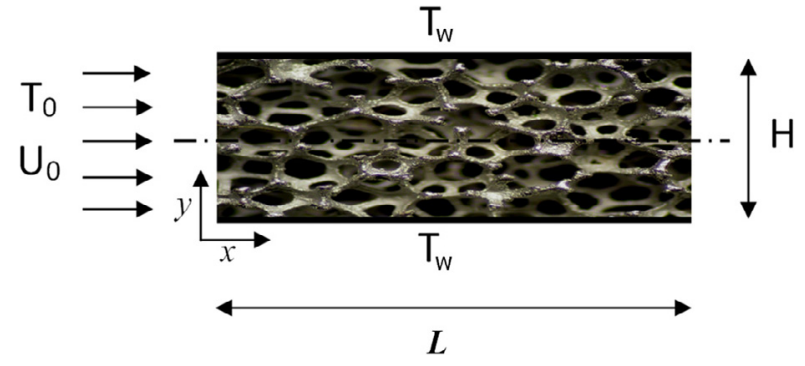

Fig. 2. Schematic diagram of the studied domain.

\subsection{Macroscopic transport equations}

The channel is considered completely filled with homogeneous and isotropic metal foam. The fluid flow is modeled using a Brinkman-Forchheimer-extended Darcy model and is assumed to be in local thermal nonequilibrium with the porous medium. The wall temperature is higher than the inlet temperature of the fluid.

\subsubsection{Flow equations}

In the porous media, the macroscopic equations obtained after volume integration over a representative elementary volume (REV) are given as:

a) Continuity

$$
\nabla \cdot \bar{u}_{D}=0
$$

b) Momentum equation

$$
\begin{aligned}
\rho \nabla\left(\frac{\bar{u}_{D} \bar{u}_{D}}{\varepsilon}\right)= & -\nabla\left(\varepsilon \bar{P}^{\mathrm{i}}\right)+\mu \nabla^{2} \bar{u}_{D} \\
& -\left[\frac{\mu \varepsilon}{K} \bar{u}_{D}+\rho \frac{C_{f} \varepsilon^{2}}{\sqrt{K}}\left|\bar{u}_{D}\right| \bar{u}_{D}\right]
\end{aligned}
$$

c) Energy equation

In this case, it is considered that the assumption of LTE is not verified. Thus, the heat transfer is governed by an energy equation for each phase.

- Solid phase

$$
0=\nabla\left(K_{e f f, s} \nabla \bar{T}_{s}\right)-h_{s f} a_{s f}\left(\bar{T}_{s}-\bar{T}_{f}\right)
$$

- Fluid phase

$$
\begin{aligned}
\left(\rho C_{p}\right)_{f}\left[\nabla\left(\bar{u}_{D} \bar{T}_{f}\right)\right]= & \nabla\left(K_{\text {eff }, f} \nabla \bar{T}_{f}\right) \\
& +h_{s f} a_{s f}\left(\bar{T}_{s}-\bar{T}_{f}\right)
\end{aligned}
$$

where $K_{\text {eff,s }}$ and $K_{\text {eff,f }}$ are the thermal conductivities for the solid and fluid phases respectively. In this work, the effective thermal conductivities of both phases are estimated by the correlation used by Calmidi et al. [18] for metal foams. They are given by:

$$
K_{e f f, s}=(1-\varepsilon) K_{s}, \quad K_{e f f, f}=\varepsilon K_{f},
$$

$h_{s f}$ is the interstitial heat transfer coefficient and $a_{s f}$ is the specific interfacial area, $\bar{u}_{D}=\varepsilon \bar{u}^{i}$ is the intrinsic volume average velocity in macroscopic description.

d) Interstitial heat transfer coefficient

The interstitial heat transfer coefficient $h_{s f}$ for packed beds is typically calculated by using the correlation given by Wakao et al. [19]. However, there is no general specific correlation for metal foams. Therefore, the following correlation developed by Zukauskas [20], is applied for copper and steel alloys metal foams (from 10 to $60 \mathrm{PPI}$ ); it is used in this work to estimate $h_{s f}$ :

$$
h_{s f}=\left\{\begin{array}{l}
0.76 \operatorname{Re}_{d}^{0.4} \operatorname{Pr}^{0.37} K_{f} / d,\left(1 \leqslant R e_{d} \leqslant 40\right) \\
0.52 R e_{d}^{0.5} \operatorname{Pr}^{0.37} K_{f} / d,\left(40 \leqslant R e_{d} \leqslant 10^{3}\right) \\
0.26 \operatorname{Re}_{d}^{0.6} \operatorname{Pr}^{0.37} K_{f} / d,\left(10^{3} \leqslant R e_{d} \leqslant 2 \times 10^{5}\right)
\end{array}\right.
$$

where $R e_{d}$ is the Reynolds number based on pore diameter.

e) Specific interfacial area

The specific area $a_{s f}$ is estimated by the correlation proposed by Zhao et al. [2].

$$
a_{s f}=3 \pi d_{f}\left[1-\mathrm{e}^{-((1-\varepsilon) / 0.04)}\right] /\left(0.59 d_{p}\right)^{2}
$$

where $d_{\mathrm{p}}$ is the pore diameter expressed generally by the correlation proposed by Calmidi as function of pore density $\omega[2]$ :

$$
d_{p}=0.0254 / \omega
$$

In order to generalize the results, the governing equations are transformed and reduced in dimensionless form by adopting the following non-dimensional variables:

$$
\begin{gathered}
X=\frac{x}{H} \quad Y=\frac{y}{H} \quad U=\frac{u}{U_{0}} \quad V=\frac{v}{U_{0}} \\
P=\frac{p}{\left(\rho_{f} U_{0}^{2}\right) / \varepsilon^{2}} \quad \theta_{(s, f)}=\frac{T_{w}-T_{(s, f)}}{T_{w}-T_{0}}
\end{gathered}
$$

where, $T_{w}-T_{0}=\Delta T_{r e f}$ is the reference difference temperature.

Then the governing equations become:

$$
\frac{\partial U}{\partial X}+\frac{\partial V}{\partial Y}=0
$$

$$
\begin{aligned}
U \frac{\partial U}{\partial X}+V \frac{\partial U}{\partial Y}= & -\frac{\partial P}{\partial X}+\frac{\varepsilon}{R e}\left(\frac{\partial^{2}}{\partial X^{2}}+\frac{\partial^{2}}{\partial Y^{2}}\right) U \\
& -\varepsilon^{2}\left[\frac{C_{F}|\vec{V}|}{\sqrt{D a}}+\frac{|\vec{V}|}{R e \cdot D a}\right] U
\end{aligned}
$$




$$
\begin{aligned}
& U \frac{\partial V}{\partial X}+V \frac{\partial V}{\partial Y}=-\frac{\partial P}{\partial Y}+\frac{\varepsilon}{R e}\left(\frac{\partial^{2}}{\partial X^{2}}+\frac{\partial^{2}}{\partial Y^{2}}\right) V \\
&-\varepsilon^{2}\left[\frac{C_{F}|\vec{V}|}{\sqrt{D a}}+\frac{|\vec{V}|}{R e \cdot D a}\right] V \\
& U \frac{\partial \theta_{f}}{\partial X}+V \frac{\partial \theta_{f}}{\partial Y}= \frac{1}{R e \cdot \operatorname{Pr}}\left(\frac{\partial^{2} \theta_{f}}{\partial X^{2}}+\frac{\partial^{2} \theta_{f}}{\partial Y^{2}}\right) \\
&+a_{s f} \frac{H^{2}}{\varepsilon^{2} d_{p}} \frac{B i}{R e \cdot \operatorname{Pr}}\left(\theta_{s}-\theta_{f}\right) \\
& 0=\frac{\partial^{2} \theta_{s}}{\partial X^{2}}+\frac{\partial^{2} \theta_{s}}{\partial Y^{2}}-a_{s f} \frac{H^{2}}{d_{p}} \frac{B i}{(1-\varepsilon) R_{k}}\left(\theta_{s}-\theta_{f}\right)
\end{aligned}
$$

$R e=\frac{\rho_{f} H U_{0}}{\mu_{f}}, R_{k}=\frac{k_{e f f, s}}{k_{e f f, f}}$ et $B_{i}=\frac{h_{s f} d_{p}}{K_{f}}$, represent respectively the Reynolds number, the solid to fluid thermal conductivity ratio and the interstitial Biot number.

\subsubsection{Dimensionless boundary conditions}

- At the inlet of channel, the fluid enters the domain with uniform velocity and temperature which is different of wall temperature.

- The downstream length of the channel is taken sufficiently long relatively to the high to ensure that fully developed conditions are applicable at the exit.

Then, the relevant dimensionless boundary conditions are summarized as follows:

$$
\begin{aligned}
& X=0,0<Y<H, U=1, V=0, \theta_{f}=\theta_{s}=1 \\
& X=L, 0<Y<H, \frac{\partial U}{\partial X}=\frac{\partial V}{\partial X}=\frac{\partial \theta_{f}}{\partial X}=\frac{\partial \theta_{s}}{\partial X}=0 \\
& Y=H, 0<X<L, U=0, V=0, \theta_{f}=\theta_{s}=0 \\
& Y=0,0<X<L, U=0, V=0, \theta_{f}=\theta_{s}=0
\end{aligned}
$$

\section{Numerical methodology}

The problem under investigation represented by the system of Equations (9) to (13) and boundary conditions (14) is solved by FVM [21]. The SIMPLE algorithm is used for the pressure-velocity coupling. The nonlinear terms in the momentum equations, due to presence of the metal foam, are treated as source terms and linearized as described in Patankar [21]. Due to the large variation of the physical quantities in certain regions of the channel, the grid distribution is non-uniform along both directions and concentrated at wall-fluid and metal foam-fluid interfaces. Convergence was monitored in terms of the relative residue, which was set to be lower than $10^{-9}$. The resulting system of algebraic equations is solved by the Line-by-Line method combined with TDMA.

The accuracy and the validity of the present calculation were checked by comparing the results with those
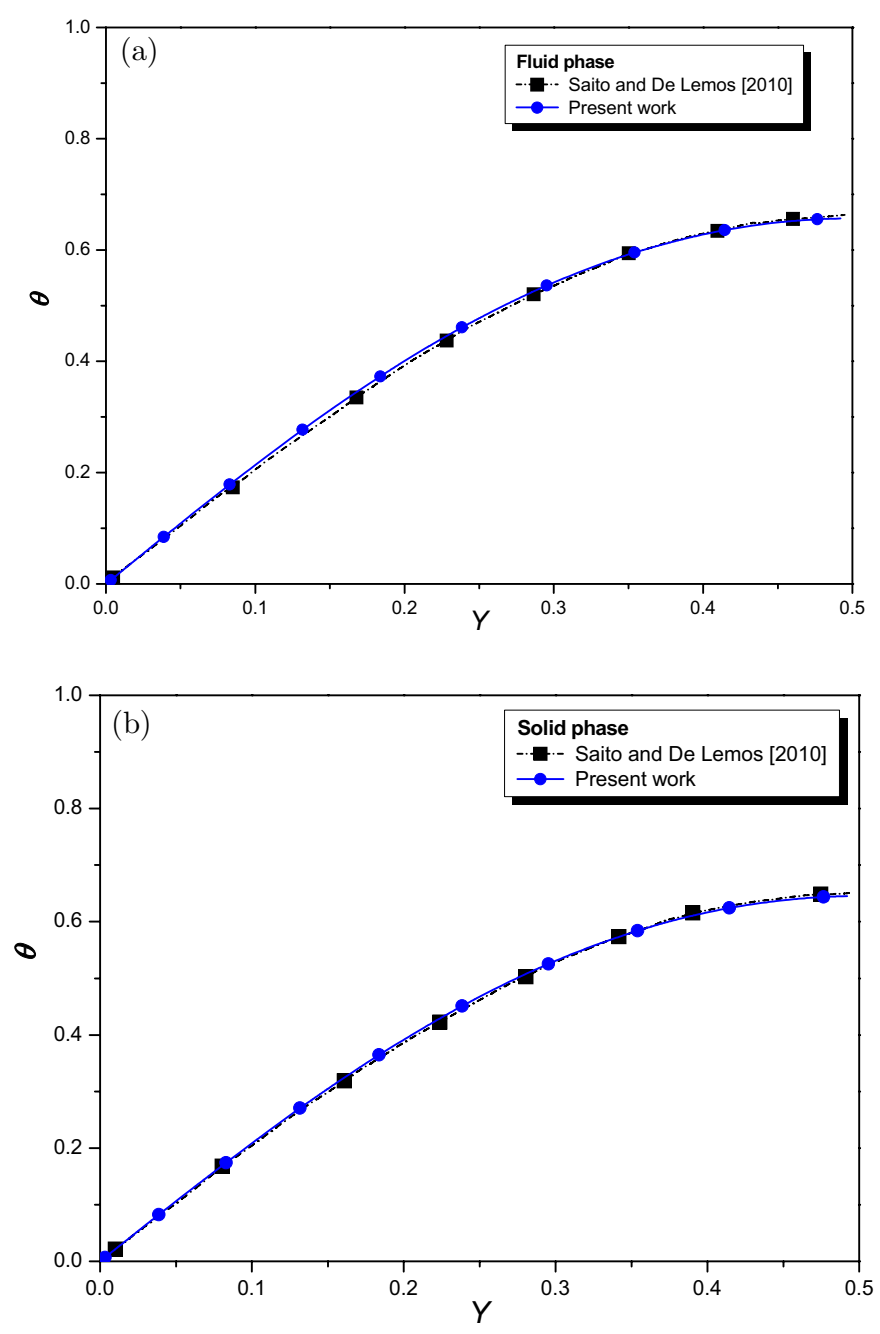

Fig. 3. Comparison of $\theta_{(s, f)}$ to the solution given by Saito and De Lemos [22]: (a) fluid phase, (b) solid phase.

of Saito and De Lemos [22], they studied the convective heat transfer for laminar flow using two-energy equation model (LTNE). The comparison of our results with those of the authors of the cited reference is obtained with the following control parameters: $D_{p} / H=0.103, R e_{d}=100$, $C f=0.55, \operatorname{Pr}=0.7, D a=10^{-4}, X=1$. The comparison is conducted with a mesh of $160 \times 80$ grids. The dimensionless temperature profiles of both solid and fluid phases are presented in Figure 3, (a) for the fluid phase and (b) for the solid one, respectively. A very good agreement is observed for all numerical comparisons.

\section{Results and discussion}

The numerical calculations are conducted for fixed characteristic values of the studied channel filled completely with metal foam matrix, presented in Table 1 . The effect of thermal conductivity ratio $R_{k}=K_{\text {eff, }, s} / K_{\text {eff }, f}$ as well as interstitial heat transfer coefficient $h_{s, f}$ is discussed and analyzed. Other parameters are studied from their variation in specific ranges. Two $R_{k}$ are chosen, the 
Table 1. Physical characteristics.

\begin{tabular}{ccccccc}
\hline$H$ & $C_{f}$ & $R e$ & $R e_{d}$ & $\varepsilon$ & $P r$ & $D a$ \\
\hline 1 & 0.07 & 580 & 100 & 0.9 & 0.7 & $10^{-4}$ \\
\hline
\end{tabular}

Table 2. $R_{k}$ for different materials used in this work.

\begin{tabular}{cccc}
\hline Material & Air & Copper & FrCrAlloy \\
\hline$K\left(\mathrm{~W} \cdot \mathrm{m}^{-1} \cdot \mathrm{K}^{-1}\right)$ & 0.0262 & 390 & 26 \\
$R_{k}=K_{\text {eff }, s} / K_{\text {eff }, f}$ & $/$ & 14885.5 & 992.37 \\
\hline
\end{tabular}

ratio of air (fluid) and two other materials often used in the manufacture of metal foams: FrCrAlloy and copper. Table 2 illustrates the thermal conductivity of the fluid (air) and the thermal conductivity of each material. The numerical results are represented especially for three transversal positions arbitrarily chosen: $X=0.1$, $X=0.9, X=2$.

In the next subsections, we present first, the effect of $R_{k}$, secondly the interstitial heat transfer coefficient, and finally, a discussion of how the metallic foam can give advantages to heat transfer process.

\subsection{Effect of the thermal conductivity ratio $R_{k}$}

In this subsection, the effect of $R_{k}$ is presented with $\theta_{s}=\theta_{f}$ in the inlet of channel. The variation of longitudinal non-dimensional temperatures is analyzed for three transversal positions: $X=0.1$ (Fig. 4a), $X=0.9$ (Fig. 4b) and $X=2$ (Fig. 4c) respectively. $R_{k}$ used, are those about FrCrAlloy and copper foams.

Figure 4 shows that the effect of $R_{k}$ on dimensionless temperature profiles is important. The non-dimensional temperature difference between Air-Copper foam and AirFrCrAlloy foam is increasing as a function of the nondimensional position $X$, and becomes large for $X=2$. Comparing to the inlet of channel, the Air-Copper foam combination approaches quickly from the wall temperature, it is due to the large difference between solid thermal conductivities of both materials. This allows to the copper foam to transmit an amount of heat greater than that obtained using FrCrAlloy foam. Thus, as $R_{k}$ is higher, much the temperature profiles are rapidly approaching from the wall temperature, also, more this phenomenon is marked, much LTE is reached quickly. This equilibrium is reached more rapidly for most conductive foams such as copper metal foam. The non-dimensional temperatures are substantially reduced at $X=2$; this diminution indicates the recovery of heat from the wall to the saturated porous medium. It means that temperature profiles tend to the wall temperature relatively to the used material. For the lower $R_{k}$ (FrCrAlloy foam), one observes that the saturated matrix by the fluid recuperates less amount of heat generated by the wall, unlike to materials with high $R_{k}$ such as copper foam. It should be noted that the difference between the temperatures of the two phases, solid and fluid, for the same materials, is very low. This is due to the significant capacity of foams in terms of
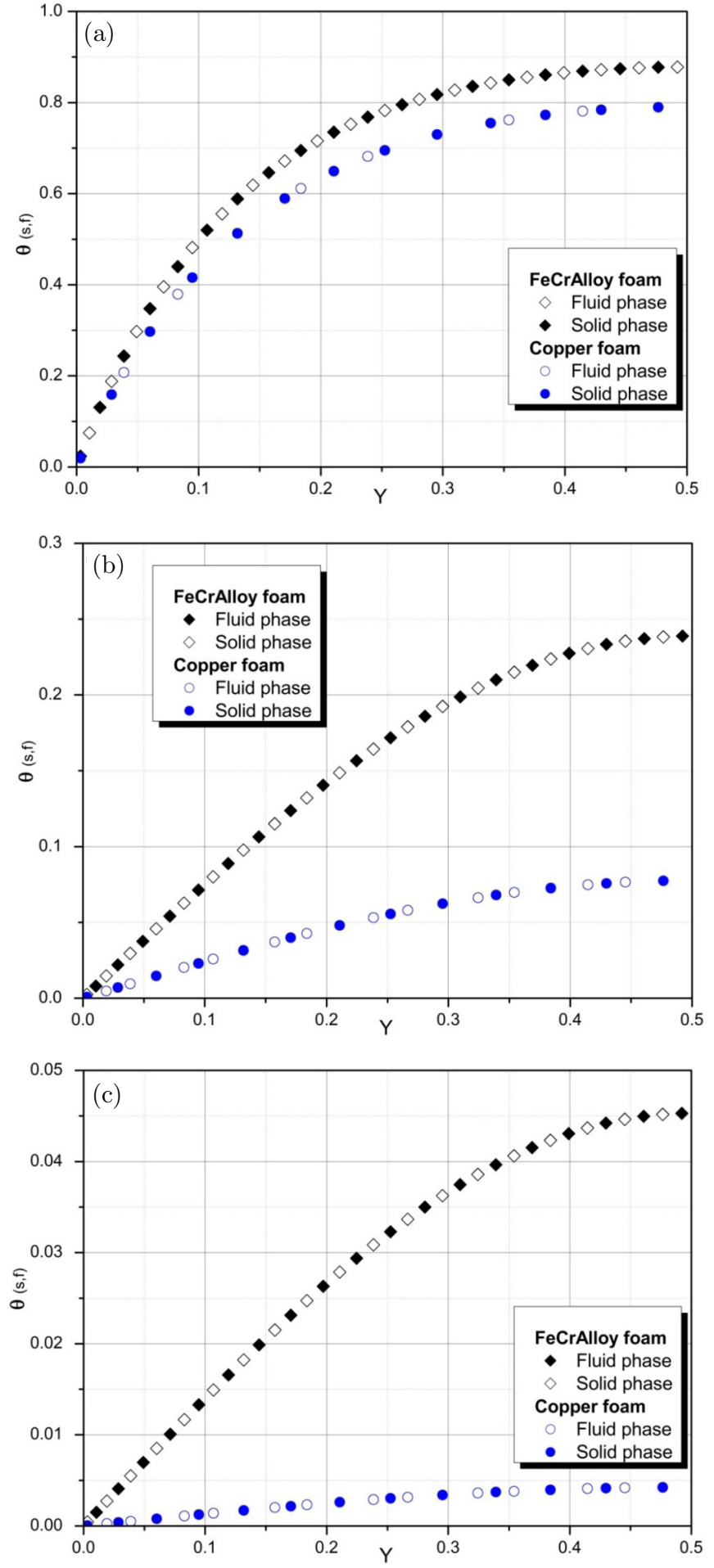

Fig. 4. Effect of $R_{k}$ on dimensionless temperature profiles: (a) $X=0.1$, (b) $X=0.9$, (c) $X=2$.

heat transfer enhancement, and to the large specific area of the metallic foams, (following to the used correlation in this work, $a_{s f}=1295 \mathrm{~m}^{2} / \mathrm{m}^{3}$ for a pore density of 10 PPI). 

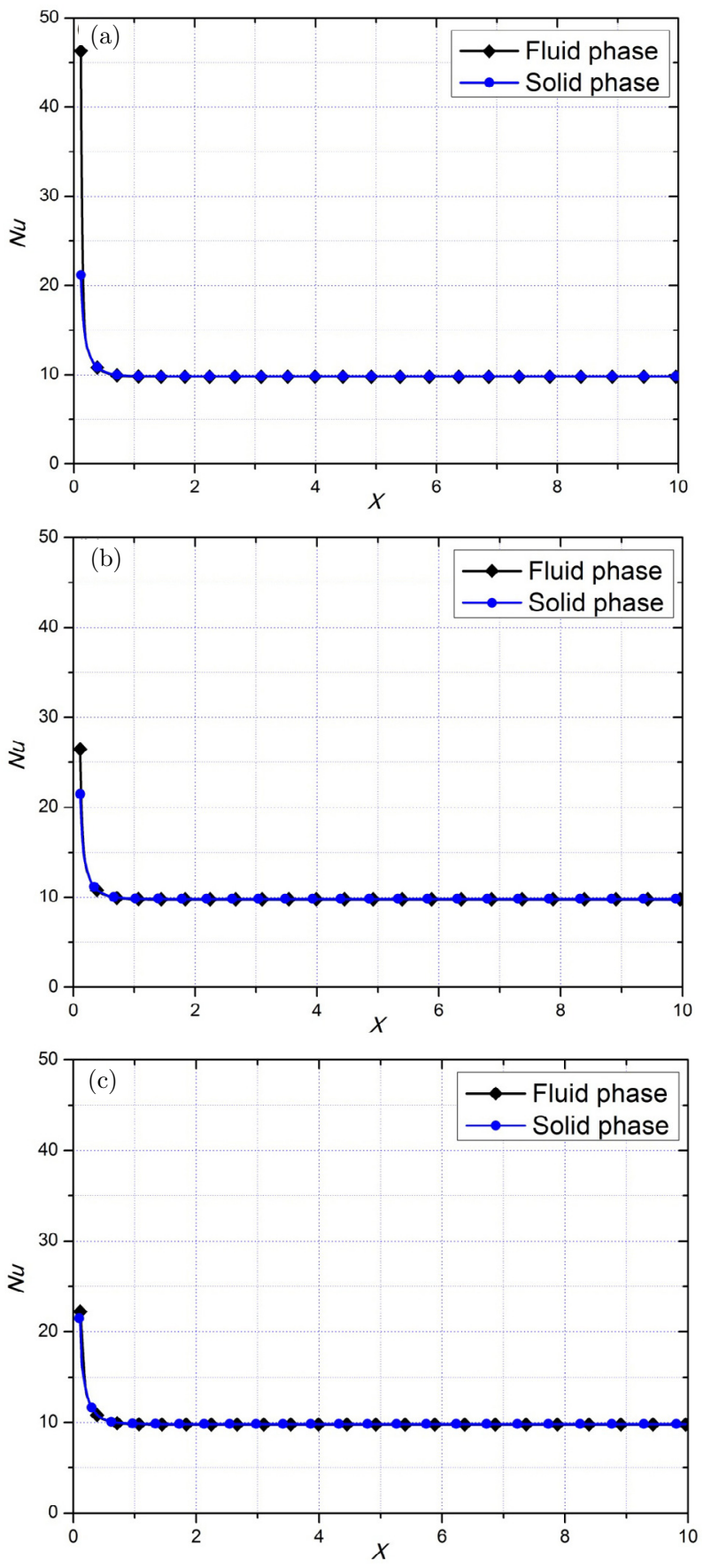

Fig. 5. Effect of $h$ on Nusselt number "FrCrAlloy foam". (a) $h_{\text {eff }}=0.01 h_{s f}$, (b) $h_{\text {eff }}=0.1 h_{s f}$, (c) $h_{\text {eff }}=h_{s f}$.

\subsection{Effect of interstitial heat transfer coefficient $h_{\text {sf }}$}

To study the effect of $h_{s f}$, a sensitivity analysis is conducted. It is expected that when $h_{s f}$ is high, the temperature difference between the fluid and solid phase becomes minimal, and LTE is reached quickly. The Nusselt number of two phases for conditions $D a=10^{-4}, R e_{d}=100$, varepsilon $=0.9, a_{s f}=1295 \mathrm{~m}^{2} / \mathrm{m}^{3}$, and $\theta_{s}=\theta_{f}$ in the inlet of the channel, is shown in Figure 5 as a function of dimensionless coordinate $X=x / H$.
In Figure 5, the nominal value of $h_{s f}$ is calculated using Zaukauskas correlation [20] which is valid for a pore Reynolds number between 40 and $10^{3}$. Effective values of $h_{s f}$ are artificially introduced by changing the nominal value: $h_{\text {eff }}=0.01 h_{s f}, h_{\text {eff }}=0.1 h_{s f}, h_{\text {eff }}=h_{s f}$. The Figure shows that the non-dimensional temperatures of solid phase are slightly lower compared to those of the fluid phase. The nominal value of $h_{s f}$ is used (Fig. 5c) and compared with two low $h_{s f}$ effective values (Figs. 5a and $5 \mathrm{~b}$ ). The local Nusselt number variation as function of $X$ is very low, except at the entrance of the channel where the difference is remarkable, particularly for the fluid phase and for lowest effective $h_{s f}$. By increasing $h_{s f}$, the convergence of two-phase curves (solid and fluid) is becoming increasingly important to the point where the two phases take the same values for large $h_{s f}$. Thus, it is found that from $X=1$, the Nusselt number takes invariable values irrespective of $h_{s f}$, this asymptotic value is equal to 9.78 for the fluid phase, and 9.83 for the solid phase. The study of $h_{s f}$ shows that the thermal equilibrium is reached rapidly for metallic foams; this, demonstrates the very significant increasing of performance in terms of heat transfer.

Note that the nominal $h_{s f}$ used in this paper is only an approximation because of the lack of a generalized correlation for this type of porous medium; however, further studies about the interstitial heat transfer coefficient would be welcome to give a better estimation.

\section{Conclusion}

The behavior of metallic foam support implemented into a flat channel was investigated. A numerical study was carried out to evaluate the following quantities: effect of solid to fluid thermal conductivity and the interstitial heat transfer. It has been shown that for the highest thermal conductivity ratios, the local thermal equilibrium is reached quickly, unlike to the lowest values, however, large values of thermal conductivity ratios, which are generally important, prove the significant contribution of these materials in terms of heat transfer, particularly by conduction. The results were used to estimate the influence of the convective contribution using metal insert structures with high porosity. It is relatively small compared to their conductive contribution between fluid and solid phases. It has been shown that such supports can bring a significant enhancement for heat transfer process due to their optimal properties.

\section{References}

[1] J. Banhart, Manufacture, characterization and application of cellular metals and metal foams, Prog. Mater. Sci. 46 (2001) 559-632

[2] C.Y. Zhao, T. Kim, T.J. Lu, H.P. Hodson, Thermal Transport Phenomena in Porvair Metal Foams and Sintered Beds, Final report, University of Cambridge, 2001 
[3] T.J. Lu, H.A. Stone, M.F. Ashby, Heat transfer in opencell metal foams, Acta Mater. 46 (1998) 3619-3635

[4] B. Madani, F. Topin, F. Rigollet, L. Tadrist, Flow laws in metallic foams: experimental determination of inertial and viscous contribution, J. Porous Media 10 (2007) 5170

[5] J. Vicente, F. Topin, Open celled material structural properties measurement: from morphology to transport properties, Mater. Trans. 47 (2006) 2195-2202

[6] B. Madani, L. Tadrist, F. Topin, Experimental analysis of upward flow boiling heat transfer in a channel provided with copper metallic foam, Appl. Thermal Eng. 52 (2013) 336-344

[7] Z.G. Qu, H.J. Xu, T.S. Wang, W.Q. Tao, T.J. Lu, Thermal Transport in Metallic Porous Media, Intech China, 2011, pp. 172-302

[8] C.Y. Zhao, W. Lu, S.A. Tassou, Thermal analysis on metal-foam filled heat exchangers, Part II: tube heat exchangers, Int. J. Heat Mass Transfer 15 (2006) 2762-2770

[9] K. Vafai, S.J. Kim, Forced convection in a channel filled with a porous medium: an exact solution, J. Heat Transfer 111 (1989) 1103-1106

[10] V.V. Calmidi, R.L. Mahajan, Forced convection in high porosity metal foams, J. Heat Transfer 122 (2000) 557565

[11] P.X. Jiang, Numerical simulation of forced convection heat transfer in porous plate channels using thermal equilibrium and non-thermal equilibrium models, Numer. Heat Transfer A 35 (1999) 99-113

[12] S. Whitaker, Improved constraints for the principle of local thermal equilibrium, Ind. Eng. Chem. Res. 30 (1991) 983-997
[13] B. Buonomo, O. Manca, G. Lauriat, Forced convection in micro-channels filled with porous media in local thermal non-equilibrium conditions, Int. J. Thermal Sci. 77 (2014) 206-222

[14] K. Yang, K. Vafai, Analysis of temperature gradient bifurcation in porous media, an exact solution, Int. J. Heat Mass Transfer 53 (2010) 4316-4325

[15] S.J. Kim, S.P. Jang, Effects of the Darcy number, the Prandtl number, and the Reynolds number on local thermal non-equilibrium, Int. J. Heat Mass Transfer 45 (2002) 3885-3896

[16] D.Y. Lee, K. Vafai, Analytical characterization and conceptual assessment of solid and fluid temperature differentials in porous media, Int. J. Heat Mass Transfer 42 (1999) 423-435

[17] N. Dukhan, Developing non thermal-equilibrium convection in porous media with negligible fluid conduction, $\mathrm{J}$. Heat Transfer 131 (2009) 014501

[18] V.V. Calmidi, R.L. Mahajan, The effective thermal conductivity of high porosity fibrous metal foams, J. Heat Transfer 121 (1999) 466-71

[19] N. Wakao, S. Kaguei, T. Funazkri, Effect of fluid dispersion coefficients on particle to fluid heat transfer coefficients in packed beds, Chem. Eng. Sci. 34 (1979) 325-336

[20] A. Zukauskas, in Convective heat transfer in cross flow, Handbook of single-phase convective heat transfer, John Wiley and Sons, New York, 1987, Chap. 6, pp. 38-41

[21] S. Patankar, Numerical heat transfer and fluid flow, CRC Press, 1980

[22] B. Saito Marcelo, J.S. de Lemos Marcelo, A macroscopic two-energy equation model for turbulent flow and heat transfer in highly porous media, Int. J. Heat Mass Transfer 53 (2010) 2424-2433 